\title{
Research on Affective State Recognition in E-Learning System by Using Neural Network
}

\author{
Egui Zhu ${ }^{1}$, Qizhen Liu ${ }^{1}$, Xiaoshuang $\mathrm{Xu}^{2}$, and Tinan Lei ${ }^{1}$ \\ ${ }^{1}$ Faculty of Education, Hubei University, Wuhan 430062, China \\ ${ }^{2}$ College of Computer Science \& Technology, Huazhong University of Science and \\ Technology, Wuhan 430000, China \\ zhuergui@yahoo.com.cn
}

\begin{abstract}
Aiming at current E-Learning system was not able to estimate the affective state of the users, an intelligent affective state recognizer that used facial expression as input was proposed and implemented in the paper. The recognizer system consists of two neural network classifiers and a fuzzy logic facial analysis module. The recognizer system had been used in an E-Learning system to recognize the emotion state. The results manifested that the recognizer system was effective. However, other bio-signals such as heartbeat, skin resistance and voice tone could also be used for affective state recognition.
\end{abstract}

Keywords: E-Learning, Neural Network, Affecting Computing, Emotion Deficiency.

\section{Introduction}

E-Learning uses modern educational technologies to implement an ideal learning environment through integrating the information technology into curriculum, which can embody the learning styles of students' main-body function, reform the traditional teaching structure and the essence of education thoroughly [1].

Although the current E-Learning systems have many merits, many of them only treat advanced information technology as simple communication tools, and release some learning contents and exercises in the network [2]. This kind of movable textbook or electronic textbook is indifferent to the learners, which lacks of the interaction of emotion. Besides, this kind of learning materials without using of the superiority of interactive multimedia technology and displaying the function of network effectively, which leads to the phenomenon of emotion deficiency in the current E-Learning system.

Emotion recognition is one of the most fundamental and important modules. It is always based on facial and audio information. At present, many scholars have carried on a great of researches on facial emotion recognition method. For example, face detection, face recognition, facial feature extraction, and some attempts at automatic facial expression analysis have been made [3] [4]. It is only recently that researchers are starting to integrate these techniques into systems that can make use of the affective state of the user. 
The goal of the work presented in this paper is the analysis of people's facial expressions from an image using a combination and extension of existing algorithms. Algorithms for face detection, feature extraction and face recognition were integrated and extended to develop a facial expression analysis system that is used in current Elearning systems. The new facial expression analysis system uses a neural network and fuzzy approach to interpret facial expressions. Although recognizing people's facial expression is a challenging task, it can lead to many exciting developments in human-computer interaction.

\section{System Framework}

Fig.1 shows the overall framework of the intelligent affective state recognizer. The affective state recognizer is composed of image Grabber, Pre-processing, classification, feature extraction, and interpretation.



Fig. 1. System Framework

The workflow of system is as follows

The image of the user is captured by a web cam which is then preprocessed by a neural network so that the position of the face in the image is identified. The next phase identifies the user based on the database of known users using principle component analysis. The final two stages consist of a facial feature extraction system based on. Neural networks and an affective state estimator based on fuzzy logic. All of the processing is completed at the client application side of the system. This means that any online implementation of this system will only send the final affective state of the user to the server side of the system providing only a small overhead. This 
chapter discusses in detail the two neural network classifiers used for face detection and facial feature location and the fuzzy logic based affective state recognizer.

\section{Key Technologies}

\subsection{Neural Network Based Face Detection}

A sixteen by sixteen pixel sub-sample of the web cam image is selected and examined to see if it is the region of the face. This is repeated for all sub-samples choosing the best candidate face for further processing. A neural network approach is adopted, in which the 256 pixel sub-sample image is given as input to a neural network that has been trained to identify face images. There are 256 neurons as input, 40 neurons in hidden layer one, 16 neurons in hidden layer two, and one output neuron. The transfer functions are tan-sigmoid functions. The training data is taken from standard face databases so that it is robust to variations in race, gender and age.

\subsection{Eye Detection}

Similar to face detection, a neural network was used to detect people's eyes. An eye database was extracted from the face database that was use for face detection. The eyes were normalized to the same size and with the iris in the centre of the eye image. A feed-forward back-propagation network was created for face detection. The network is a 3 layers-structure. It has 2 hidden layers and 1-output layer. It also has 1 layer for input. Each eye image is 18 pixels in width by 12 pixels in height forming a rectangular image. So, there are 216 neurons as input, 8 neurons in hidden 216 inputs 8 hidden neurons 4 hidden neurons 1 output layer 1,4 neurons in hidden layer 2 , and 1 output neuron. The transfer functions are tan-sigmoid function. Using this approach the exact position of the eyes in the image can be identified as well as the candidate point for the eyebrows since they are invariably above the eye for an upright full frontal image of the face.

\subsection{Mouth Detection}

Mouth detection is challenging because of the difficulty in modeling the shape of mouth. Firstly, the lighting of the mouth region is corrected locally. Secondly, noise in the mouth region of the image is eliminated using a wavelet transformation algorithm. Thirdly, Sobel edge detection combined with Laplacian edge detection is used followed by a morphing logic operation to fill in the holes. The largest blob found by this procedure is the mouth. Once the mouth has been identified candidate points for the mouth outline are available[5] [6].

\section{Conclusion}

An intelligent affective state recognizer that used facial expression as input was proposed in the paper. The facial expression analysis system implemented is based on a fuzzy approach. Six expressions, namely, happiness, anger, surprise, sadness, puzzled 
and disgusted are detected. Fig. 2 shows the affective state recognition software. The bars in the boxes to the right of the image show the result of the intelligent expression analysis. The longest bar is the best value and if it is significantly larger the other remaining values a conclusive decision on the affective state of the user can be estimated.

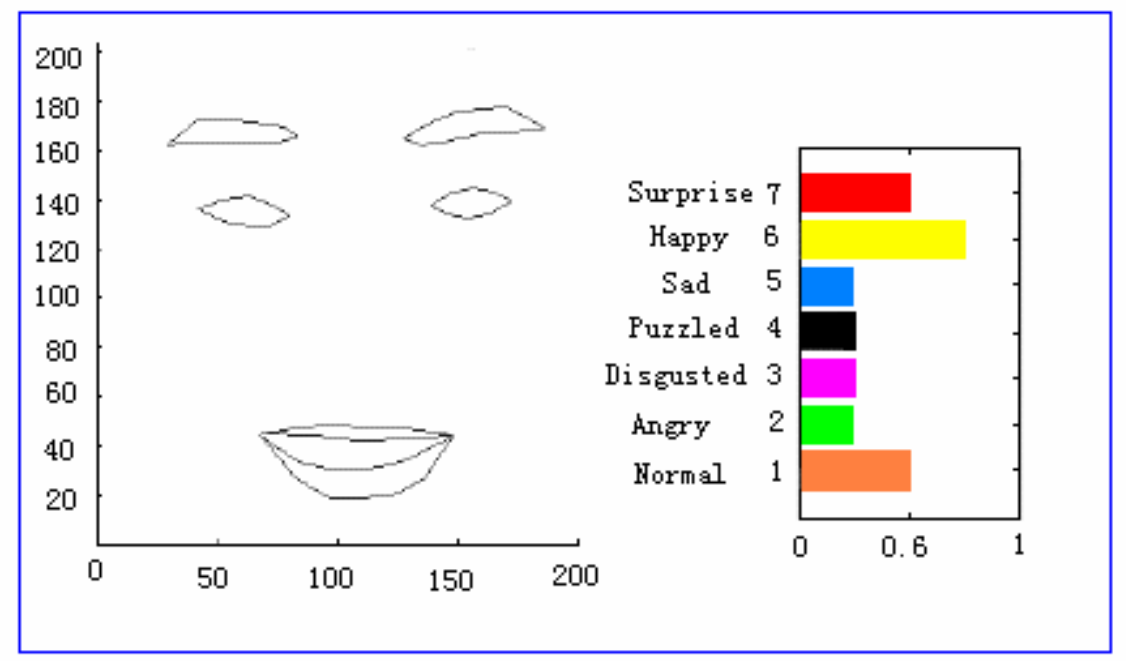

Fig. 2. Affective State Recognition Software

\section{References}

1. He Kekang.: E-Learning Essence- information technology into curriculum. E-education Research, 105, 1, 2002, 3-4.

2. Wang Jijun.: Emotion deficiency and compensation in distance learning. Chinese network education, 2005.

3. R.W.Picard.: Affective Computing. Cambridge. MIT Press, 1997.

4. R.W.Picard.: Affective Computing, Challenges. Cambridge. International Journal of Human Computer studies, 59(1), 2003, 55-64.

5. X. Li and Q. Ji.: Active Affective State Detection and User Assistance with Dynamic Bayesian Networks. Proceedings of Bayesian Modeling Applications Workshop, 2003.

6. M. Pantic and L.J.M. Rothkrantz.: Automatic Analysis of Facial Expressions, the State of the Art. IEEE Transactions on Pattern Analysis and Machine Intelligence, vol. 22, 2000, 1424-1445. 